\title{
Studies on Molecular Composite V. Effects of Introducing Side Chains into an Aromatic Polyamide on the Phase Structure of a Polymer Blend Material Comprising the Aromatic Polyamide as a Reinforcing Polymer
}

\author{
Hiroshi Akita and Hiroto Kobayashi \\ Fundamental Research Division, Wako Research Center, Honda R\&D Co., Ltd., \\ Chuo, Wako-shi 351-0193, Japan
}

(Received February 17, 1999)

\begin{abstract}
KEY WORDS Nano Composite / Precursor Polymer/Polymer Blend / 3-D Network Structure / Aromatic Polyamide /
\end{abstract}

Molecular composites and nano composites are polymer blend materials consisting of a reinforcing polymer with a rigid molecular structure dispersed on a nanometer level in a flexible matrix polymer. ${ }^{1,2}$ Because of excellent mechanical properties, ${ }^{3,4}$ Poly ( $p$-phenylene benzobisthiazole) (PBZT) should be useful as reinforcing material for molecular composites or nano composites. ${ }^{5-8}$ We synthesized a precursor polymer of $\operatorname{PBZT}^{9,10}$ and investigated applications as reinforcing material in molecular composites and nano composites. $^{11-13}$ The polymer was an aromatic polyamide with organic side chains which could be removed by $350^{\circ} \mathrm{C}$ heat treatment which was followed by the formation of five-membered rings of PBZT. As an example, a matrix polymer of thermoplastic aromatic polyamide and a reinforcing polymer of a random copolymer consisting of the PBZT precursor and fragment in common with the matrix polymer was blended and then molded for the obtaining PBZT nano composite materials. Two nano composites with different phase structures were obtained due to varied bulk molding process conditions. One bulk specimen was formed by uniformly dispersed PBZT particles with approx. 30 nanometers in diameter in the matrix polymer, and another specimen formed by a three-dimensional (3-D) network structure of PBZT fibrils with 30-60 nanometer diameter was obtained. ${ }^{13}$ Mechanical properties and heat resistance of the nano composite are improved considerably due to the formation of the fine 3-D network structure of rigid-rod reinforcing polymer fibrils, even with small amounts of the reinforcing element, in contrast to other type of PBZT composite materials. ${ }^{13}$

Generally, polymer blending using a small quantity of a rod-like reinforcing polymer and large quantity of a flexible matrix polymer gives a microphase separation structure in which microdomains of the rod-like polymer are dispersed in the matrix polymer. The origins of the unique phase structures appears to be not only the effects of the molding process, including the thermal isomerization of the precursor polymer to PBZT, but also specialized blended materials. The PBZT precursor polymer is an all-para-type aromatic polyamide which has thioether side chains. Introducing the bulky side chains may influence liquid crystallinity in polymer blend solutions and phase structure of consolidated polymer blend materials. The matrix polymer was a thermoplastic aromatic polyamide which have similar molecular structure to that of the PBZT precursor polymer, so that inducing a strong intermolecular hydrogen bridges between the matrix polymer and reinforcing polymer to give fine dispersibility of the reinforcing element was expected.

We investigated the influence of introducing the side chains to the reinforcing polyamide on mechanical properties and phase structure of blended materials, in order to search for molecular design of reinforcing polymer materials and matrix polymer utilized for another polymer nano composite materials, by means of blending or copolymerizing of aromatic, half-aromatic or aliphatic polyamide matrix polymers and the PBZT precursor polymer.

\section{EXPERIMENTAL}

\section{Materials}

The PBZT precursor monomer, an aramid matrix polymer and PBZT random copolymer were synthesized according to procedures described in a previous report. ${ }^{11,12} \mathrm{~A}$ random copolymer comprising an aromatic polyamide without side chains was synthesized using paraphenylenediamine (Tokyo-Kasei) in place of the PBZT precursor monomer. We used 3-chloro-terephthalic acid chloride (3-CTC, Tokyo-Kasei) an acid chloride monomer substituted with one $\mathrm{Cl}$ atom, to improve the solubility of obtained precursor polymers in organic solvent.

A block copolymer consisting of the PBZT precursor and a half-aromatic polyamide was synthesized as follows. The PBZT precursor monomer $(3.6 \mathrm{mmol})$ was dissolved in $10 \mathrm{ml} 5 \mathrm{wt} \% \mathrm{LiCl} / N$-methyl-2-pyrrolidone (NMP) in argon atmosphere and cooled in an ice bath. The solution was added 3-CTC $(3.95 \mathrm{mmol})$ and stirred for $30 \mathrm{~min}$ to synthesize oligomer. 4-Aminophenylacetic acid $(0.33 \mathrm{mmol}$, Tokyo-Kasei) was added to the reaction solution to introduce carbonyl terminal group to the oligomer. NMP (28.6 mL), pyridine $(21.5 \mathrm{~mL})$ and $\mathrm{LiCl}$ $(2.5 \mathrm{~g})$ were dissolved in the solution and adipic acid (10.85 mmol), 3,3'-diaminodiphenylmethane $(10.85 \mathrm{mmol})$ and triphenylphosphine $(31.6 \mathrm{~g})$ were added. The solution was stirred for $6 \mathrm{~h}$ at $100^{\circ} \mathrm{C}$ to induce copolymerization. The resulting polymer was coagulated by pouring the solution into excess methanol, then washed and dried 
under vacuum. A block copolymer consisting of aromatic polyamide without side chains and half-aromatic polyamide was synthesized using paraphenylenediamine in place of the PBZT precursor monomer.

Homopolymer of the PBZT precursor was synthesized as described in a previous report. ${ }^{11}$ An aromatic polyamide homopolymer without side chains was synthesized using paraphenylenediamine in place of the PBZT precursor monomer. 6-Nylon (Aldrich) was purchased and used without further purification.

Polymer blending using aromatic polyamide matrix polymer was performed as described in a previous report. ${ }^{13}$ Bulk specimens with $80 \times 15 \times 2$ millimeter size were obtained by consolidating the polymer blend powder at $230^{\circ} \mathrm{C}$ in a closed mold with $9.8 \mathrm{MPa}$ of pressure.

Molding of the block copolymer comprising halfaromatic polyamide was performed at $230^{\circ} \mathrm{C}$ in a closed mold with $5.9 \mathrm{MPa}$ of pressure.

Polymer blending using aliphatic polyamide matrix polymer was performed as follows. The reinforcing polymer $(3.0 \mathrm{~g})$ and $\mathrm{LiCl}(3.0 \mathrm{~g})$ were dissolved in $50 \mathrm{~mL}$ of NMP. To the solution was added 6-Nylon $(7.0 \mathrm{~g})$ and stirred for $3 \mathrm{~h}$ at $160^{\circ} \mathrm{C}$. The polymer blend material was coagulated by pouring into excess $\mathrm{MeOH}$, washed and dried under vacuum followed by molded at $220^{\circ} \mathrm{C}$ with $9.8 \mathrm{MPa}$ of pressure.

\section{Measurements}

Flexural properties of the consolidated specimen were determined using a universal test machine (Shimadzu AG-5000) in the three-point bending test at $25^{\circ} \mathrm{C}$. The phase structure of the polymer blend samples was observed using a transmission electron microscope (TEM, Philips CM-120 STEM-TWIN) with accelerating voltage of $80 \mathrm{kV}$ without staining. Elementary analysis of phases was done using energy dispersive X-ray spectra (EDX, EDAX DX-4) combined with the TEM.

\section{RESULTS AND DISCUSSION}

\section{Aromatic Polyamide Matrix Polymer and PBZT Precursor Copolymer}

A thermoplastic aromatic polyamide was blended as a matrix polymer with two random copolymers consisting of all-para aromatic polyamides and a fragment in common with the matrix polymer. Polymers used in this case are shown in Figure 1. A thermoplastic polyamide " $3,4^{\prime}$-I" with $0.51 \mathrm{dL} \mathrm{g}^{-1}$ (in NMP, $30^{\circ} \mathrm{C}$ ) of inherent viscosity was used as the matrix polymer and random copolymers consisting of all-para aramid and a fragment in common with the matrix polymer with a partial molar ratio of 8:2 were used. ${ }^{12,13}$ The PBZT precursor and paraphenylenediamine were used as all-para aromatic moieties. Inherent viscosity of the random copolymer comprising the PBZT precursor was $1.05 \mathrm{dL} \mathrm{g}^{-1}$ and that of the random copolymer consisting of an aromatic polyamide with no side chains was $0.93 \mathrm{dL} \mathrm{g}^{-1}$. Molding of the blended material was carried out at $230^{\circ} \mathrm{C}$. At this temperature, the conversion of the precursor to PBZT was little and ignored. ${ }^{9,10}$

Flexural modulus of the bulk specimens obtained by blending these polymers are indicated in Table I against the weight percent of the reinforcing polymers in the bulk specimens. The theoretical tensile modulus of the PBZT precursor polymer is inferior to those of the aromatic polyamide with the same main chain and no side chains, because of increased molecular diameter. Introducing the bulky thioether side chains to the all-para aromatic polyamide main chain restricts intermolecular packing so that the aggregation and orientation of the rigid-rod molecular chains are also restricted. Generally, these effects spoil mechanical properties of the aromatic polyamide. However, restriction of aggregation of the rigid-rod polymer in polymer blend solutions and consolidated polymer blend material improves dispersibility of the reinforcing polymer and changes its phase structure to more suitable for reinforcement. These effects will improve the mechanical properties of polymer blend nano composite materials. Differences of molecular structures between these reinforcement polymers influ-
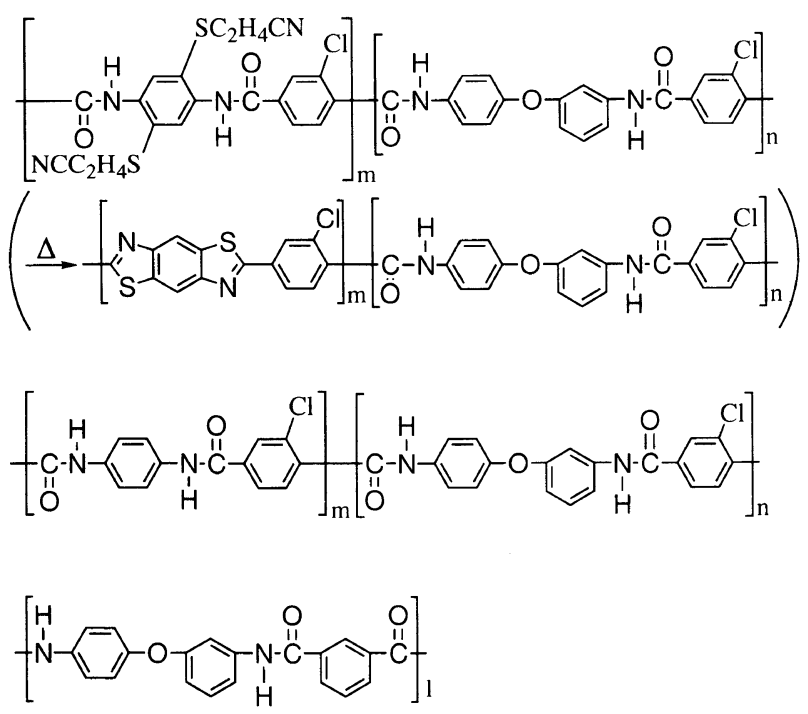

Figure 1. Molecular structures of random copolymers consisting of flexible aramid and PBZT precursor (upper) or all-para aromatic polyamide with no side chains (middle), and 3,4'-I matrix polymer (lower).

Table I. Flexural properties of bulk specimens obtained by blending 3,4'-I flexible aramid matrix homopolymer and two types of random copolymers comprising all-para aromatic polyamide

\begin{tabular}{lcl}
$\begin{array}{c}\text { Aromatic diamine for } \\
\text { synthesizing the random copolymer }\end{array}$ & Amount of PBZT precursor & Ft $\%$ \\
\hline $\begin{array}{l}\text { PBZT precursor diamine } \\
\text { para Type aromatic diamine with no } \\
\text { side chains }\end{array}$ & 30 \\
\hline
\end{tabular}


ence crystallinity of the matrix polymer of the polymer blend materials. The effect was ignored in this study since the difference in the reinforcement effect resulting from the difference in the dispersion of all-para aromatic polyamide was assumed far greater than the effect of crystallization of the matrix polymer.

The modulus indicated much higher reinforcing effect of the random copolymer comprising the PBZT precursor than that of the random copolymer consisting of an aromatic polyamide with no side chains. The phase structure of these bulk specimens could not be determined because of the similarity of the molecular structure between the aramid matrix and precursor polymer, even when using TEM, but the high mechanical potential suggested a desirable phase structure generating high reinforcement efficiency of the reinforcing copolymer, such as continuous phase, to be formed based on restriction of aggregation of the rigid-rod polymer as a result of introducing side chains into the all-para aromatic polyamide main chain. The modulus of the polymer blend material obtained from random copolymer comprising the PBZT precursor was higher than that of heat converted rigid-rod PBZT nano composite with uniformly dispersed PBZT particles in the matrix polymer. ${ }^{13}$ It was much lower than that of nano composite with PBZT 3-D network structure in the matrix polymer, ${ }^{13}$ and the PBZT precursor constructed continuous phase but orientation of the PBZT precursor may thus be restricted by the bulky thioether side chains. Because of the polymer blended bulk specimen obtained from $3,4^{\prime}-\mathrm{I}$ and PBZT precursor copolymer was identical to the intermediate product of the 3-D network PBZT nano
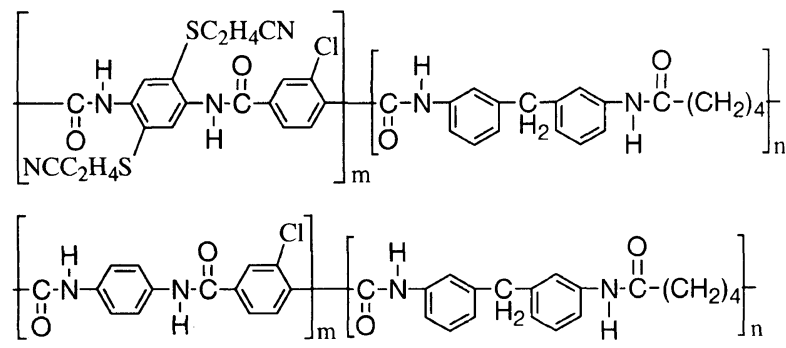

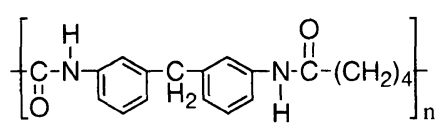

Figure 2. Molecular structures of block copolymers consisting of half-aromatic polyamide and PBZT precursor (upper) or all-para aromatic polyamide with no side chains (middle), and matrix polymer (lower). composite, this mechanism is related to the construction of the 3-D network structure of the PBZT nano composite obtained by heat treatment of the PBZT precursor-blended specimen at $350^{\circ} \mathrm{C}$ to convert the precursor to PBZT. ${ }^{14}$

\section{Half-Aromatic Polyamide Matrix Polymer and PBZT Precursor Block Copolymer}

A thermoplastic half-aromatic polyamide was blended as a matrix polymer with two block copolymers consisting of all-para aromatic polyamides and a fragment in common with the matrix polymer. Polymers used in this case are shown in Figure 2. Inherent viscosity of the half-aromatic polyamide matrix polymer was $0.42 \mathrm{dL} \mathrm{g}^{-1}$. Block copolymers consisting of all-para aramid and a fragment in common with the matrix polymer with a partial molar ratio of $8: 2$ were used as reinforcing material in the nano composite. ${ }^{12,13}$ Inherent viscosity of the block copolymer comprising the PBZT precursor was $1.17 \mathrm{dL} \mathrm{g}^{-1}$ and that of the block copolymer consisting of an aromatic polyamide with no side chains $1.51 \mathrm{dL} \mathrm{g}^{-1}$. Molding was carried out at $230^{\circ} \mathrm{C}$.

Flexural modulus of the bulk specimens are indicated in Table II against the weight percent of rigid-rod moiety in the bulk specimens. The modulus of polymer blend materials comprising the block copolymer comprising the PBZT precursor was much higher than that of the copolymer consisting of aromatic polyamide with no side chains.

PBZT block copolymers consisting of the PBZT precursor oligomer and flexible half-aromatic polyamide oligomer could be molded without blending with matrix polymer to give bulk specimens. In the case of a block copolymer consisting of the PBZT precursor and aromatic polyamide, its phase structure and mechanical properties were likely to be similar to those of polymer blended materials consisting of PBZT and the aromatic polyamide matrix polymer. ${ }^{13}$ Flexural modulus of the bulk specimens obtained are indicated in Table III against the weight percent of rigid-rod moiety in the bulk specimens. The modulus of the molded block copolymer comprising of the PBZT precursor was much higher than that of the copolymer consisting of aromatic polyamide with no side chains.

\section{Aliphatic Polyamide Matrix Polymer and the PBZT Precursor}

Because the aliphatic polyamide has a highly different molecular structure from that of the PBZT precursor, it

Table II. Flexural modulus of bulk specimens obtained by blending half-aromatic polyamide matrix homopolymer and block copolymers consisting of half-aromatic polyamide and PBZT precursor or all-para aromatic polyamide with no side chains for various partial molar ratios of aromatic polyamides

\begin{tabular}{lcc}
\hline $\begin{array}{c}\text { Aromatic diamine used for } \\
\text { synthesizing the block copolymer }\end{array}$ & Amount of PBZT precursor & Flexural modulus \\
\cline { 2 - 3 } & wt $\%$ & 30 \\
PBZT precursor diamine & 40 \\
para Type aromatic diamine with & 30 & 9.10 \\
no side chains & 40 & 7.13 \\
\end{tabular}


Table III. Flexural modulus of bulk specimens obtained by molding block copolymers consisting of half-aromatic polyamide and PBZT precursor or all-para aromatic polyamide with no side chains for various partial molar ratios of aromatic polyamides

\begin{tabular}{|c|c|c|}
\hline \multirow{2}{*}{$\begin{array}{l}\text { Aromatic diamine used for } \\
\text { synthesizing the block copolymer }\end{array}$} & Amount of PBZT precursor & Flexural modulus \\
\hline & $\mathrm{wt} \%$ & $\mathrm{GPa}$ \\
\hline \multirow[t]{3}{*}{ PBZT precursor diamine } & 20 & 5.74 \\
\hline & 30 & 9.10 \\
\hline & 40 & 9.19 \\
\hline \multirow{2}{*}{$\begin{array}{l}\text { para Type aromatic diamine with } \\
\text { no side chains }\end{array}$} & 20 & 5.15 \\
\hline & 30 & 7.12 \\
\hline
\end{tabular}

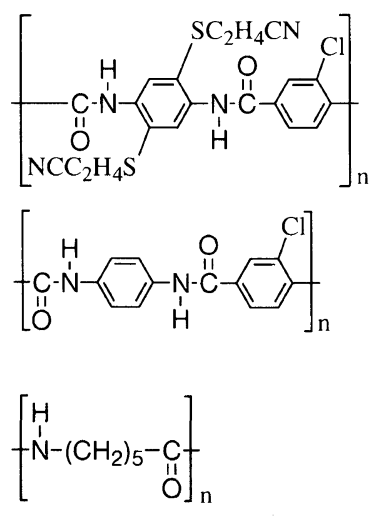

Figure 3. Molecular structures of PBZT precursor homopolymer (upper), all-para aromatic polyamide without side chains (middle) and 6-Nylon matrix polymer (lower).

is very difficult to obtain a polymer blend sample comprising these polymers with fine dispersibility. Takayanagi et al. to blended poly( $p$-phenylene terephthalamide) (PPTA), an all-para type of aromatic polyamide with no side chains, with 6-Nylon by codissolving both polymers in sulfuric acid followed by rapid coagulation using a coagulation bath for restricting macro phase separation of the polymers. ${ }^{14}$ The obtained polymer blend material was comprised of microfibrils of PPTA with 20 of L/D and a 15-30 nanometer diameter. The PPTA microfibrils were dispersed in 6-Nylon matrix polymer with no continuous phase.

6-Nylon was blended as a matrix polymer with two homopolymers consisting of all-para aromatic polyamides. Polymers used in this section are shown in Figure 3 . Inherent viscosity of the PBZT precursor homopolymer was $1.05 \mathrm{dL} \mathrm{g}^{-1}$ and that of the homopolymer consisting of an aromatic polyamide with no side chains, $1.13 \mathrm{dL} \mathrm{g}^{-1}$. Flexural modulus of the bulk specimens obtained are plotted in Figure 4 against the weight percent of the reinforcing polymer in the bulk specimens. The modulus of the blended material comprising the PBZT precursor was much higher than that of the polymer blend consisting of aromatic polyamide with no side chains. The phase structure of the bulk specimen consisting of PBZT precursor polymer was observed by TEM and is shown in Figure 5. The results of EDX elemental analysis of each phase are shown in Figure 5. The PBZT prepolymer consists of more atoms, such as sulfur and chlorine (see Figure 3), compared to the matrix polyamide. The bulk specimen had two phase structures consisting of a Nylon-6 matrix-rich spherical phase

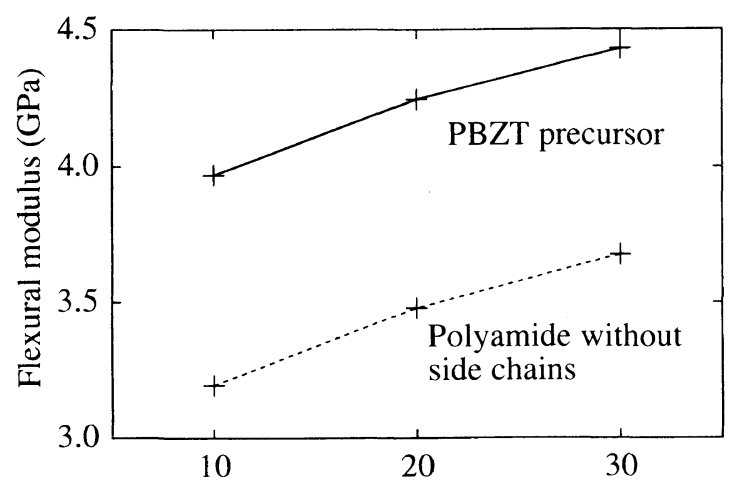

Partial molar ratio of PBZT precursor or all-para aromatic polyamide without side chains $(\%)$

Figure 4. Relationship of flexural modulus of bulk specimens obtained by blending 6-Nylon aliphatic polyamide matrix polymer and PBZT precursor or all-para aromatic polyamide with no side chains for various partial molar ratios of aromatic polyamides.

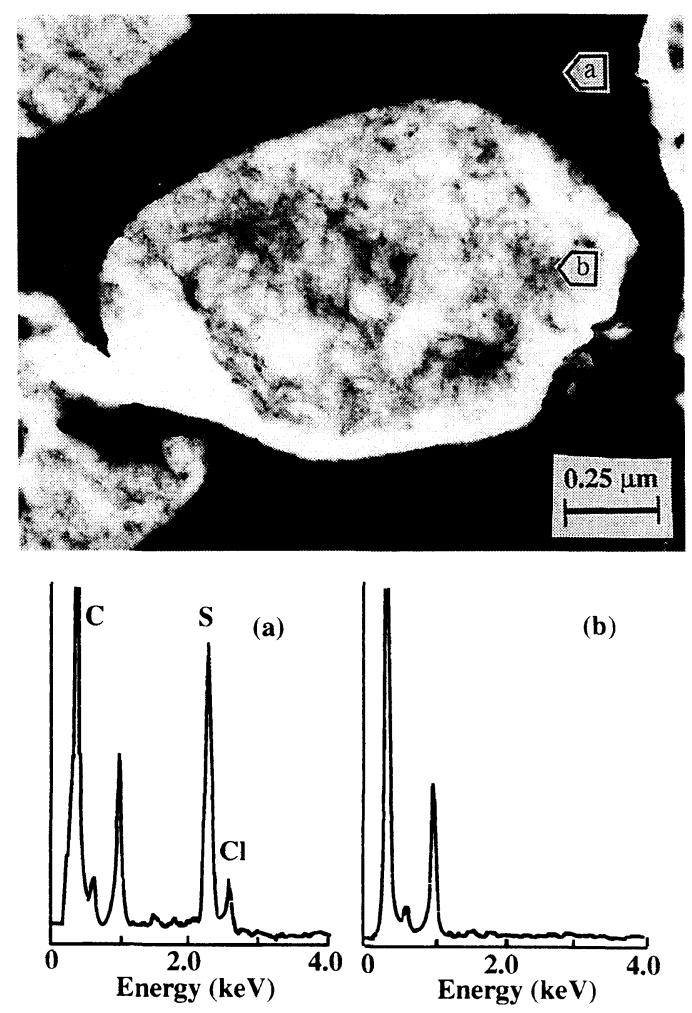

Figure 5. TEM image (upper) and EDX spectra (lower) for each phase (a, PBZT prepolymer-rich phase; $b, 6$-Nylon-rich phase) of phase structure of bulk specimen consisting of PBZT precursor polymer and 6-Nylon matrix polymer. Dark regions are PBZT prepolymers.

Polym. J., Vol. 31, No. 10, 1999 
dispersed in PBZT prepolymer-rich continuous phase. Because of great differences in molecular structure between the aliphatic polyamide matrix polymer and PBZT precursor, size of the phase structure was much larger than that of the nano composite materials consisting of the PBZT random copolymer and aromatic polyamide matrix polymer. The structure was the reverse image of that of a reported phase structure obtained by polymer blending of a rigid-rod polymer such as PPTA ${ }^{14}$ or rigid-rod PBZT with 6-Nylon matrix polymer. ${ }^{8}$

In this study, in spite of spoiled mechanical properties of the aromatic polyamide with thioether side chains introduced into the all-para aromatic polyamide main chain, stronger reinforcing effect of aromatic polymer comprising the PBZT precursor was observed than that of aromatic polymer with no side chains. In one case, a unique continuous phase structure comprising the PBZT precursor was observed. This suggests the possibility of controlling liquid crystallinity in polymer blend solutions and micro phase structure of consolidated polymer blend materials such as the molecular composite or the nano composite by introducing bulky side chains into the reinforcing polyamide. This may serve as basis for molecular design of polymer materials utilized for another polymer nano composite materials such as a functional polymer composite material.

\section{REFERENCES}

1. M. Hasegawa, M. Kochi, R. Yokota, and I. Mita, Eur. Polym. J., 25, 349 (1989).

2. M. Takayanagi, Pure Appl. Chem., 55, 819 (1983).

3. S. R. Allen, R. J. Farris, and E. L. Thomas, J. Mater. Sci., 20, 2719 (1985)

4. S. R. Allen, R. J. Farris, and E. L. Thomas, J. Mater. Sci., 20, 2727 (1985).

5. H. Chuah, C. Y. Lee, and T. E. Helminiak, "Molecular Composites Processing and Properties I," Air Force Wright Aeronautical Laboratories, US-Government Printing Office, AD-A194 113, 1988, pp 41-59.

6. K. N. Ptak, K. M. Gupte, C. Y-C. Lee, and H. H. Chuah, "Polymeric Materials Science and Engineering," New Orleans, Louisiana, U.S.A., 1987, p 517.

7. T. Nishihara, H. Mera, and K. Matsuda, Proc. ACS Polymeric Materials Science and Engineering Proceedings, Vol. 55, American Chemical Society, Washington, D.C., 55, 1986, p 821.

8. H. H. Chuah, T. Kyu, and T. E. Helminiak, Polymer, 30, 1591 (1989).

9. T. Hattori, H. Akita, M. Kakimoto, and Y. Imai, Macromolecules, 25, 3351 (1992).

10. T. Hattori, H. Akita, M. Kakimoto, and Y. Imai, J. Polym. Sci., Part A, Polym. Chem., 30, 197 (1992).

11. H. Akita, and T. Hattori, J. Polym. Sci., Part B, Polym. Phys., 37, 189 (1999)

12. H. Akita, H. Kobayashi, T. Hattori, and K. Kagawa, J. Polym Sci., Part B, Polym. Phys., 37, 199 (1999).

13. H. Akita, and H. Kobayashi, J. Polym. Sci., Part B, Polym. Phys., 37, 209 (1999).

14. M. Takayanagi and T. Ogata, J. Macromol. Sci., Phys., B17, 591 (1980). 